\title{
An enhanced modulation signal bispecturm analysis for bearing fault detection based on non-Gaussian noise suppression
}

\author{
Junchao Guo ${ }^{1}$, Hao Zhang ${ }^{1}$, Dong Zhen ${ }^{1,}$, Zhanqun Shi ${ }^{1}$, Fengshou Gu ${ }^{2}$, Andrew. D. Ball ${ }^{2}$ \\ ${ }^{1}$ School of Mechanical Engineering, Hebei University of Technology, Tianjin, 300401, China. \\ ${ }^{2}$ Centre for Efficiency and Performance Engineering, University of Huddersfield, Huddersfield, HD1 3DH, UK. \\ ${ }^{*}$ Corresponding author: E-mail address: d.zhen@hebut.edu.cn (D. Zhen)
}

\begin{abstract}
Many methods have been developed for machinery fault diagnosis addressing only Gaussian noise reduction, the major weaknesses of these methods are their performance for non-Gaussian noise suppression. Modulation signal bispectrum (MSB) is a useful signal processing method with significant advantages over traditional spectral analysis as it has the unique properties of Gaussian noise elimination and demodulation. However, it is susceptible to non-Gaussian noise that normally occurs in the practical applications. In view of the deficiency of MSB, in this paper, an autoregressive (AR) modeling filter was developed based on non-Gaussian noise suppression to improve the performance of MSB for machinery fault detection. The AR model was considered as a pre-filter process unit to reduce the non-Gaussian noise. And the order of the AR model, which can affect the performance of the AR filter, was determined adaptively using a kurtosis-based indicator. However, the existing nonlinear modulation components remain in the AR filtered signal. The MSB was then applied to decompose the modulated components and eliminate the Gaussian noise from the filtered signal using AR model for the fault feature extraction accurately. The advantage of the AR model can effectively manage non-Gaussian noise, whereas the MSB can suppress Gaussian noise and is illustrated in the simulation signal. Furthermore, the proposed AR-MSB method was applied to analyze the vibration signals of defective bearings with outer race and inner race faults. By comparison, the proposed approach had a superior performance over conventional MSB and fast kurtogram in extracting fault features and was precise and effective for rolling element bearing fault diagnosis.
\end{abstract}

Keywords: Non-Gaussian noise; Autoregressive modeling; Modulation signal bispectrum; Rolling element bearings 


\section{Introduction}

Rolling element bearings, as common transmission components in the mechanical equipment, are among the most vulnerable components. The health status of the bearing will directly affect the working performance and operating life of the mechanical equipment [1-2]. Thus, the rolling element bearing fault detection is of great significance for carrying out maintenance activities [3-4]. When a local fault occurs in the rolling element bearings, the harmonic frequency components caused by the fault defect will interact with the resonant frequency of the system [5]. The spectrum obtained by the Fourier transform is more affected by the resonant frequency of the system, and the important fault information is covered because of undetectable high-order harmonic mixing [6]. Therefore, demodulating the vibration signal in the high frequency mode can eliminate the confusion caused by heavy noise or other high frequency components.

Envelope analysis (EA), as a typical signal demodulation analysis method, has been developed for mining modulation information, which has been widely applied in the field of rolling element bearing fault diagnosis [7-8]. However, the center frequency and bandwidths of the bandpass filters need to be determined in advance. Fast kurtogram (FK) was originally proposed by Antoni et al. [9] to automatically determine the optimal filter center and bandwidth of the bandpass filter, overcoming the shortcomings of manually determining the parameters of the bandpass filter. In recent years, increasing attentions have been paid to the advantages of using FK in the fault diagnosis of rotating machinery [10-13]. However, the FK reveals weaknesses when there are strong random noise and interfering frequencies in the original vibration signals. Recently, modulation signal bispectrum (MSB) has been applied to mining modulation information because it has the advantages of demodulating the modulated components and decomposing interference frequency components of the signals, which was introduced by Gu et al. [14]. With its advantage, MSB has attracted extensive attention in the fault diagnosis of mechanical systems. For example, Zhang et al. applied the MSB to monitor the extent of gear wear deterioration [15]. Tian et al. proposed an MSB-based analysis method that can bring more precise and robust detection results than FK in a range of representative scenarios [16]. Guo et al. developed a hybrid analysis method based on wavelet packet energy (WPE) and MSB to diagnose faults in rotating machinery [17]. And Guo et al. put forward a new multi-stage noise reduction method combined with ensemble empirical mode decomposition (EEMD), wavelet thresholding (WT) and MSB to detect rolling element bearing faults [18]. These research works demonstrated the advanced performance of MSB in demodulation and noise reduction. However, Gaussian noise obeying normal distribution is usually employed to evaluate the noise reduction performance of the developed methods, non-Gaussian noise, such as the random noise obeying uniform distribution, Weibull distribution and Rayleigh distribution etc. [19], is limited to be considered for the advanced signal processing methods development.

Generally, the Gaussian noise is universal in nature and engineering, and has been extensively investigated in fault diagnosis and processing, while the non-Gaussian noise has not obtained enough attention up to now [20-21]. In fact, the non-Gaussian noise cannot be neglected as it plays an important role in the effect on the fault diagnosis results. A few researchers proved that non-Gaussian noise is the main component of the background noise in the field of machinery fault diagnosis. Wang et al. introduced a maximum correntropy unscented Kalman filter (MCUKF) into non-Gaussian noise [22]. Asadi et al. applied a new method using entropy estimation of eigenvalues to estimate Gaussian noise and nonGaussian noise in complex vibration signals [23]. Yen et al. put forward a novel approach based on radical basis function (RBF) and recurrent networks to extract weak transient signal in non-Gaussian noise, and 
the performance of his method is validated using both simulation and experiment signals [24]. These methods have shown that the non-Gaussian noise is very significant in fault diagnosis and cannot be ignored. However, MSB was developed based the assumption that the noise is Gaussian type. This means that it is not very effective in suppressing non-Gaussian noise in theory, which was also investigated in [25]. Consequently, MSB probably show poorer performance for enhancing fault features by analyzing the signals polluted by strong non-Gaussian noise.

To further enhance the performance of MSB, an AR filter was developed based on the AR model analysis to eliminate the non-Gaussian noise in this paper. The AR model has been validated high performance in removing non-Gaussian noise and is widely used in fault detection [26-27]. The AR model can be considered as a pre-filter that used to improve the signal-to-noise ratio (SNR) of the raw signal through suppressing the non-Gaussian noise. And the kurtosis-based indicator was used to determine the optimal order of the AR model. Then the MSB was applied to the denoised signal provided by the AR model to decompose the modulated components and eliminate the Gaussian noise, thus can extract the fault features accurately for fault detection. The proposed method was applied to diagnose the rolling element bearing faults based on the simulation and experimental studies. A comparison between the proposed method, FK and conventional MSB has been conducted to evaluate the performance of identifying the rolling element bearing faults.

The paper is organized into 6 sections. After the introduction, Section 2 covers the basic concept of AR model and its order optimization with respect to the periodic impulse signals. Section 3 introduces the theoretical basis of the MSB. Section 4 demonstrates the comprehensive implementation of the proposed method, denoted as AR-MSB, and is validated based on different simulated signals. Section 5 applies the proposed AR-MSB approach to analyze the experimental signals for diagnosing rolling element bearings with the inner and outer race faults, respectively. Section 6 draws the conclusions based on the analysis results of the studies.

\section{AR modeling filter}

As the parameters of the AR model contain the important state characteristics of the system and are very sensitive to the state changes of the system, it has great value for time series analysis. Moreover, the AR model works extremely well in terms of capturing the key features of any data series. Literatures on damage detection using the AR model are quite extensive [28-29]. And a linear AR model can be expressed as [30]:

$$
X_{m}=\sum_{i=1}^{p} a_{i} X_{m-i}+\varepsilon_{m}
$$

where $X_{m}$ and $p$ are the predicted signal value at the time $m$ and the order of the model, $a_{i}(i=$ $0,1,2, \ldots, p)$ represents the weighting coefficients. The $\varepsilon_{m}$ is the error term, indicating the difference between the actual and linear predicted value. It includes additional white noise and non-stationarities in the forms of impulses [31]. Therefore, the AR model is able to suppress the non-Gaussian noise of a signal through removing the error terms $\varepsilon_{m}$ based on an AR prediction filter.

The block diagram for the AR prediction filter design is shown in Fig. 1 [32], where $X_{p}$ is the $p^{\text {th }}$ data point predicted by the AR filter and $X_{m-p}$ is the pre-filtered signal after interception. The model coefficients can be estimated using different methods. The two most common methods are autocorrelation algorithms and least squares algorithms [33]. In this paper, the least squares algorithm is mainly used for the parameter estimation. 


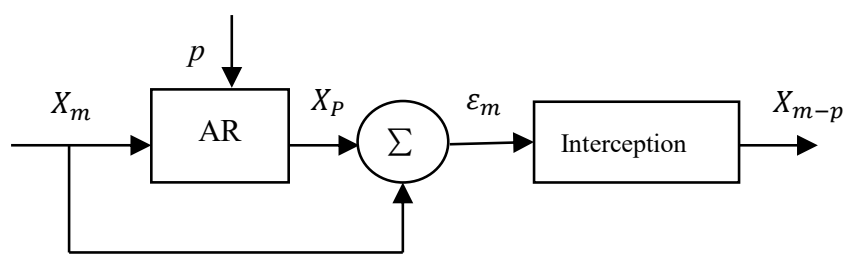

Fig. 1. The process of AR prediction filtering.

At present, some relevant specific criteria's have already been applied in the AR model order selection. For example, Akaike's information criterion (AIC) [34], final prediction error criterion (FEP) [33], Bayesian information criterion (BIC) [35] and Variational Bayesian (VB) [36]. In [32] the maximum kurtosis was proposed to optimum AR model order due to the signal containing both impulses and stationary noise. Besides, the maximum kurtosis has been certified to be an effective method for selecting the optimal order [37-38]. Therefore, the maximum kurtosis was applied to optimize the optimal order of the AR model in this paper.

\section{Modulation signal bispectrum}

The MSB is an advanced signal processing technique based on the improvement of the conventional bispectrum. According to the theoretical analysis, MSB not only has the characteristics of conventional bispectrum that is able to vanish for Gaussian processes, but also can demodulate the modulated components which are inherent in the measured signals [25]. Therefore, the MSB is applied to inhibit the Gaussian noise and extract the modulated components of the measured vibration signal for bearing fault detection in this study.

For a discrete-time signal $x(t)$ with corresponding discrete Fourier transform $X(f)$, the MSB can be defined in the frequency domain as [14]:

$$
B_{M S}\left(f_{1}, f_{2}\right)=E<X\left(f_{1}+f_{2}\right) X\left(f_{1}-f_{2}\right) X^{*}\left(f_{1}\right) X^{*}\left(f_{1}\right)>
$$

where $B_{M S}\left(f_{1}, f_{2}\right)$ and $E<>$ mean the bispectrum of signal $x(t)$ and expectation operator. And $f_{1}$ and $f_{2}$ denote the carrier frequency and modulating frequency, $\left(f_{1}+f_{2}\right)$ and $\left(f_{1}-f_{2}\right)$ represent the higher and lower sideband frequencies, respectively. If $x(t)$ is a Gaussian signal, its $B_{M S}\left(f_{1}, f_{2}\right)=$ 0 . It indicates that MSB offers high noise immunity when the vibration signal is mixed with Gaussian noise [25].

The overall phase of the MSB is written as below:

$$
\emptyset_{M S}\left(f_{1}, f_{2}\right)=\emptyset\left(f_{1}+f_{2}\right)+\emptyset\left(f_{1}-f_{2}\right)-\emptyset\left(f_{1}\right)-\emptyset\left(f_{1}\right)
$$

When two components $f_{1}$ and $f_{2}$ are coupled, their phases are defined by the following relationships:

$$
\left\{\begin{array}{l}
\varnothing\left(f_{1}+f_{2}\right)=\varnothing\left(f_{1}\right)+\emptyset\left(f_{2}\right) \\
\varnothing\left(f_{1}-f_{2}\right)=\varnothing\left(f_{1}\right)-\emptyset\left(f_{2}\right)
\end{array}\right.
$$

By substituting Eq. (4) into Eq. (3), it indicates that the whole phase of the MSB is zero and the amplitude of the MSB is calculated through the product of the four magnitudes, representing the maximum of the complex product. Therefore, a bispectral peak appears at bifrequency $\left(f_{1}, f_{2}\right)$. The Eq. (2) consists of the $\left(f_{1}+f_{2}\right)$ and $\left(f_{1}-f_{2}\right)$ to measure the nonlinearity of the modulated components. If $\left(f_{1}+f_{2}\right)$ and $\left(f_{1}-f_{2}\right)$ are both because of nonlinear effect between $f_{1}$ and $f_{2}$, a bispectral peak appears at bifrequency $\left(f_{1}, f_{2}\right)$. Therefore, it is more extractive and distinctive in denoting the modulation characteristics in a noisy signal.

To more accurately quantize the sideband amplitude, the MSB is improved by magnitude 
normalization to remove the effect of carrier frequency of $f_{1}$. The MSB sideband estimator (MSB-SE) is expressed as [18]:

$$
B_{M S}^{S E}\left(f_{1}, f_{2}\right)=\frac{B_{M S}\left(f_{1}, f_{2}\right)}{\sqrt{\left|B_{M S}\left(f_{1}, 0\right)\right|}}
$$

where $B_{M S}\left(f_{1}, 0\right)$ means the squared power spectrum estimation at $f_{2}=0$.

To get more robust results, the MSB detector is modified by the average of a few suboptimal MSB slices:

$$
B\left(f_{2}\right)=\frac{1}{N} \sum_{n=1}^{N} B_{M S}^{S E}\left(f_{1}^{n}, f_{2}\right),\left(f_{2}>0\right)
$$

where $N$ denotes the whole number of the chose $f_{1}$ suboptimal slices, which relies on the significance of the peaks themselves.

To obtain suboptimal $f_{1}$ slices, $B_{M S}^{S E}\left(f_{1}^{n}, f_{2}\right)$ is denoted by the compound MSB slice $B\left(f_{1}\right)$, which is computed by averaging the significant MSB peaks in the incremental direction of the $f_{2}$ [18]:

$$
B\left(f_{1}\right)=\frac{1}{M-1} \sum_{m=2}^{M} B_{M S}^{S E}\left(f_{1}, m \Delta f\right)
$$

where $\Delta f$ represents the frequency resolution in the $f_{2}$ orientation. In summary, the flowchart of the MSB detector is displayed in Fig. 2.

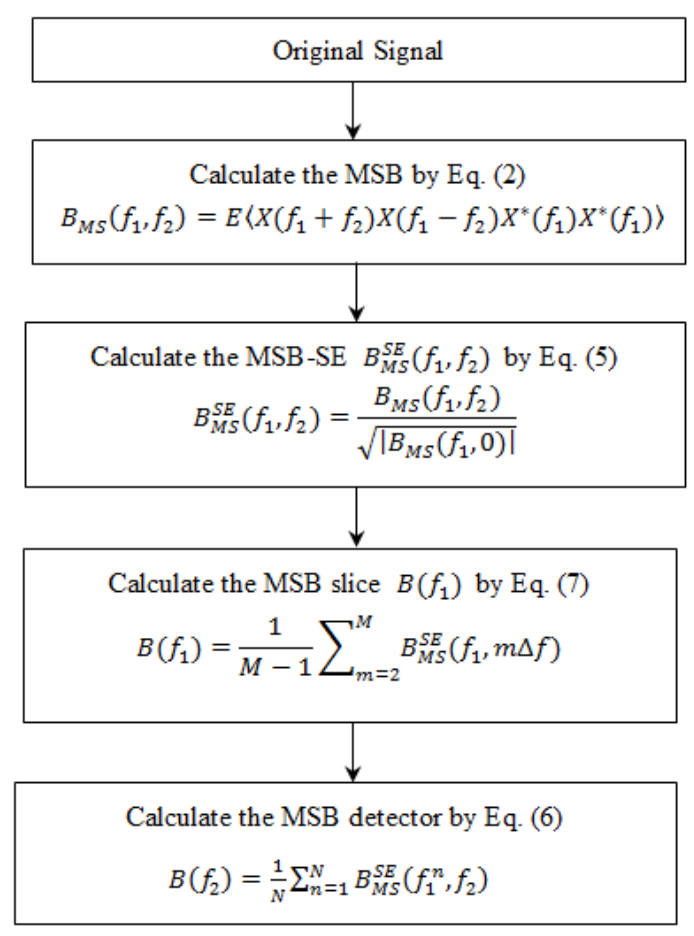

Fig. 2. The flowchart of the MSB detector.

\section{The proposed AR-MSB method}

\subsection{The AR-MSB diagnosis scheme}

Motivated by the advantages of AR model and MSB, this paper proposed the AR-MSB for fault diagnosis of rolling element bearings. The AR model can effectively eliminate the non-Gaussian noise in the vibration signal, and its non-Gaussian noise mainly includes random noise obeying uniform distribution, Weibull distribution and Rayleigh distribution, etc. Among them, the Rayleigh distribution is the most widely used due to its simplicity and saving computation time [39-40]. Thus, the Rayleigh 
distribution random noise was selected as the non-Gaussian noise for the simulation study in this paper. The MSB has the characteristics of removing the Gaussian noise and demodulating the modulated components which are inherent in the measured signals. The structural framework of the proposed method is demonstrated in Fig. 3 and summarized as follows:

Step 1: Determine an appropriate order range of the AR model. The general range for the order $p$ can be setup from 1 to 100 [38].

Step 2: Apply the least squares algorithm to determine the weighting parameter $a_{i}(i=0,1,2, \ldots, p)$ of the AR model under different orders $p$, and calculate the kurtosis values of the error terms $\varepsilon_{m}$.

Step 3: Select the order $p$ that corresponding to the maximum kurtosis value as the optimal AR model order.

Step 4: Calculate the MSB of the AR filtered signal using Eq. (2).

Step 5: Calculate the MSB sideband estimator $B_{M S}^{S E}\left(f_{1}, f_{2}\right)$ by Eq. (5).

Step 6: Calculate the compound MSB slice $B\left(f_{1}\right)$ by Eq. (7).

Step 7: Calculate the MSB detector $B\left(f_{2}\right)$ using Eq. (6) to extract the fault characteristic frequencies.

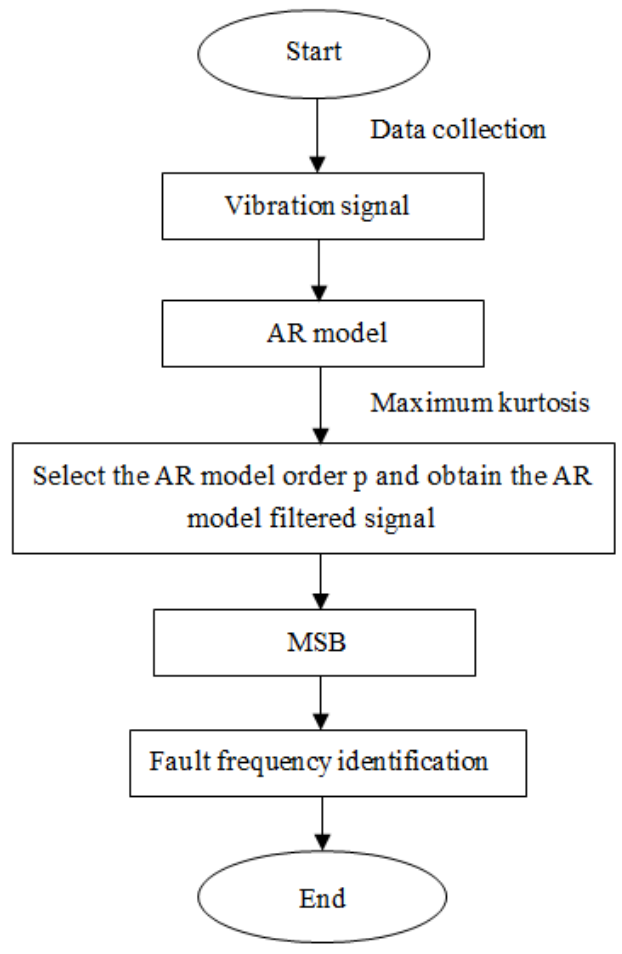

Fig. 3. The flowchart of the AR-MSB.

\subsection{Simulated study}

To verify the feasibility and effectiveness of the AR-MSB method, a bearing outer race fault simulation signal $x(t)$ can be calculated by the following formula:

$$
\left\{\begin{array}{c}
x(t)=\sum_{n=-N}^{N} A_{m}\left(t_{i}\right) e^{-\alpha\left(t_{i}\right)} \sin \left(2 \pi f_{c} t\right)+n_{1}(t)+n_{2}(t) \\
t_{i}=t-\left(m / f_{0}\right)
\end{array}\right.
$$

where $A_{m}$ denotes the amplitude of the $m^{\text {th }}$ fault impulse signal, $N$ means the number of impulses, $f_{0}=88.5 \mathrm{~Hz}$ stands the fault characteristic frequency, $\alpha$ and $f_{c}$ indicate the decay parameter and fault excited frequency, $n_{1}(t)$ shows the typical Gaussian noise with normal distribution, $n_{2}(t)$ indicates the non-Gaussian noise with Rayleigh distribution. The waveforms and probability distribution function (PDF) of $n_{1}(t)$ and $n_{2}(t)$ were shown in Fig. 4 and 5, respectively. 

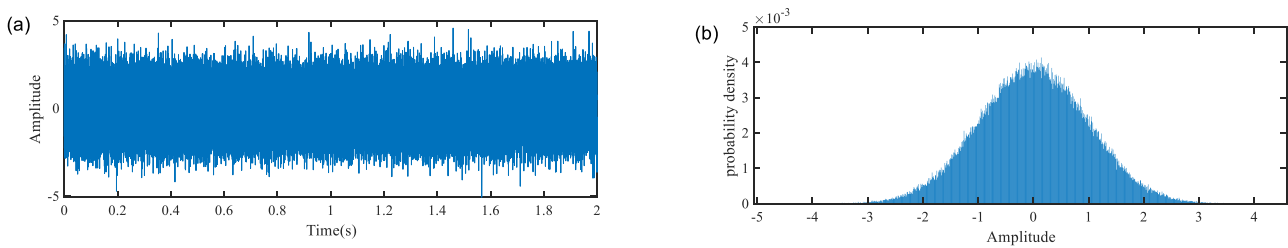

Fig. 4. The Gaussian noise with normal distribution: (a) waveform (b) PDF.
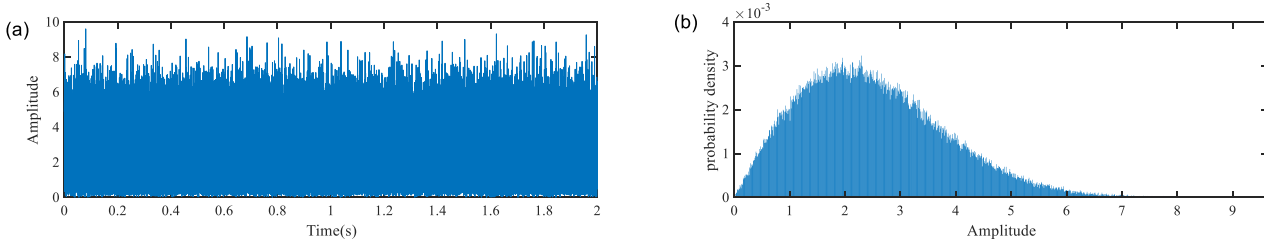

Fig. 5. The non-Gaussian noise with Rayleigh distribution: (a) waveform (b) PDF.

\subsubsection{Non-Gaussian noise reduction}

Fig. 6 represents the waveform and spectrum of the simulated outer race fault signal plus Gaussian noise with a SNR of $-9.62 \mathrm{~dB}$. To validate the ability of AR model for eliminating non-Gaussian, the nonGaussian noise with different SNRs was added into the simulation signal and processed by the proposed AR-MSB and conventional MSB. Fig. 7 illustrates the waveform and spectrum for the simulated signals under different SNR cases.
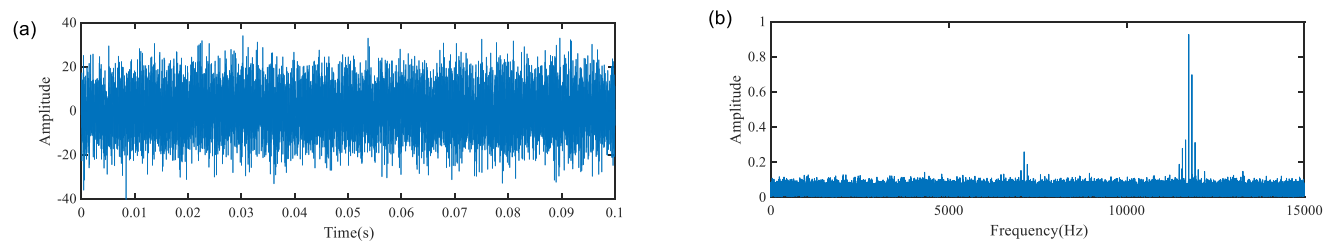

Fig. 6. Simulated signal of an outer race fault: (a) waveform (b) spectrum.
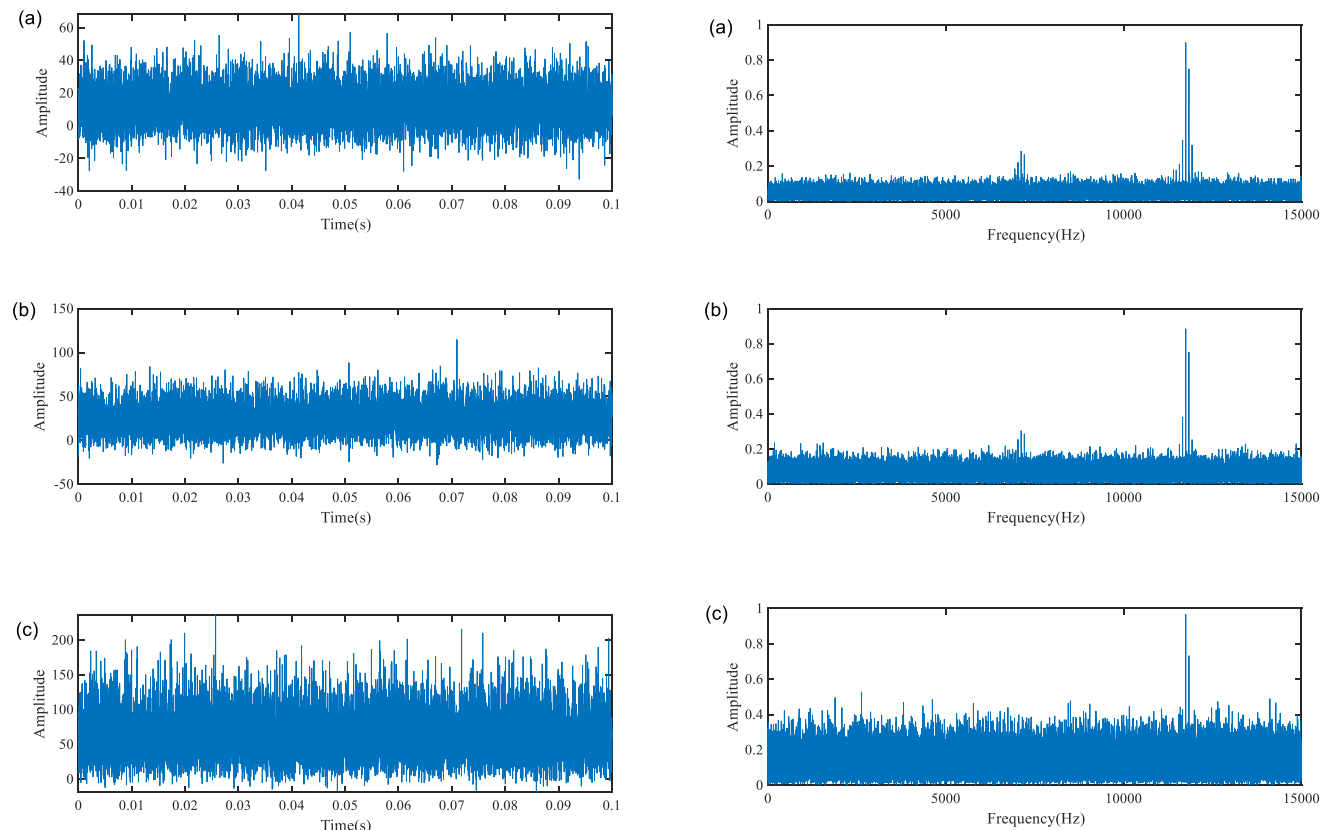

Fig. 7. Waveform and spectrum of the simulated signal with non-Gaussian noise for different SNRs: 
(a) $\mathrm{SNR}=-7.79 \mathrm{~dB}$, (b) SNR $=-10.79 \mathrm{~dB}$, and (c) $\mathrm{SNR}=-13.82 \mathrm{~dB}$.

The simulated signals were then processed using the AR model filter. According to the maximum kurtosis criterion, the optimal order $p$ and the corresponding maximum kurtosis value can be determined based on the analysis results in Fig. 8. The optimal AR model order and its corresponding maximum kurtosis value for the three SNR cases are 7 and 3.029, 6 and 3.035, 6 and 3.026, respectively.
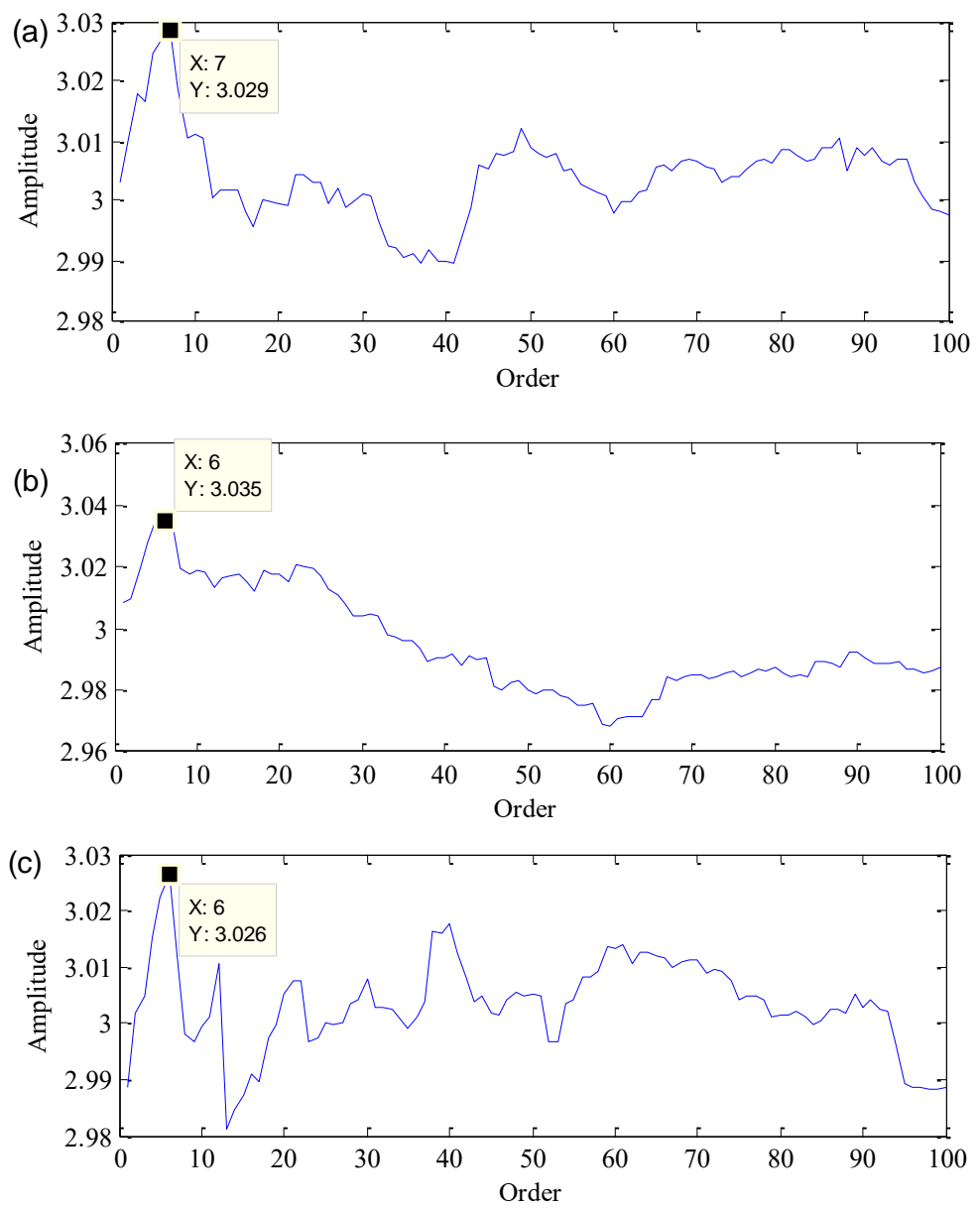

Fig. 8. Optimum AR model order and the related maximum kurtosis under different SNRs:

(a) $\mathrm{SNR}=-7.79 \mathrm{~dB}$, (b) SNR $=-10.79 \mathrm{~dB}$, and (c) $\mathrm{SNR}=-13.82 \mathrm{~dB}$.

The MSB was then applied to analyze the AR model filtered signal to decompose the modulated components. The steps of calculating the MSB are as follows: First, the MSB and MSB-SE were computed by Eq. (2) and (5). Second, MSB slice $B\left(f_{1}\right)$ was computed by Eq. (7) and suboptimal $f_{1}$ slices were selected. Finally, the MSB detector was computed by Eq. (6), and the analysis results were plotted on the left side of Fig. 9. Additionally, the conventional MSB method was used for comparison and its analysis results were displayed on the right side of Fig. 9. It can be seen that as the non-Gaussian noise gradually increasing, the effect of the fault feature extraction of the MSB becomes increasingly worse. This indicates that the MSB is very sensitive to non-Gaussian noise. Simultaneously, adding the AR model under the same conditions improved the extraction efficiency of the MSB on the left side of Fig. 9. The results demonstrated that the AR model filter can suppress non-Gaussian noise effectively.
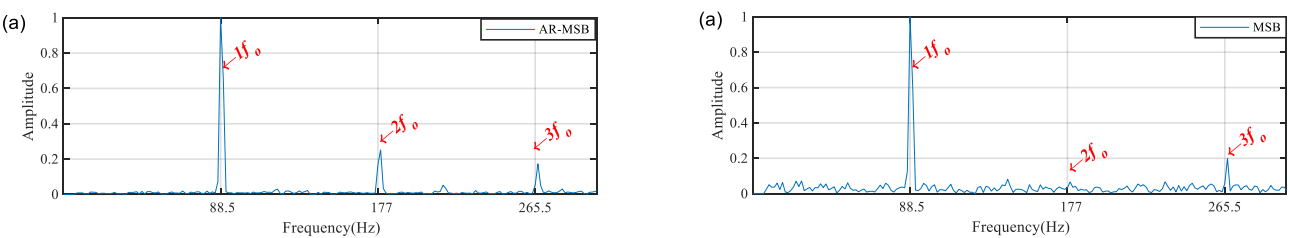

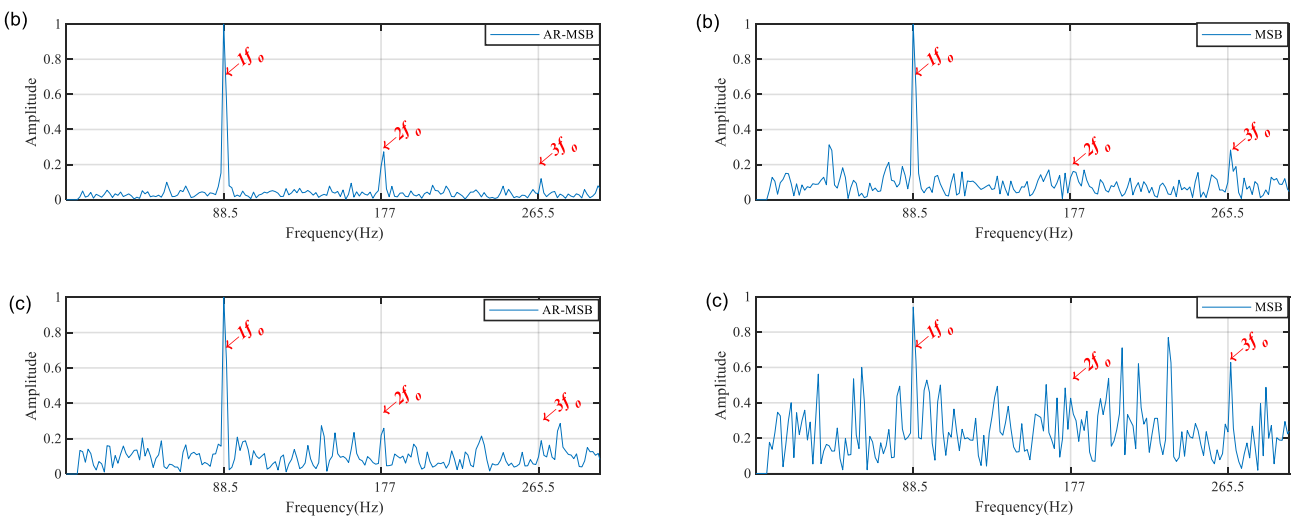

Fig. 9. The analysis results of the AR-MSB and conventional MSB for non-Gaussian noise reduction: (a) $\mathrm{SNR}=-7.79 \mathrm{~dB}$, (b) $\mathrm{SNR}=-10.79 \mathrm{~dB}$, and (c) $\mathrm{SNR}=-13.82 \mathrm{~dB}$.

\subsubsection{Gaussian noise reduction}

The waveform and spectrum of the simulated signal adding the non-Gaussian noise with the SNR of -7.80 dB was shown in Fig. 10. To validate the ability of the MSB to reduce Gaussian noise, the Gaussian noise were added into the simulated signals with different SNRs. Fig. 11 presents the waveform and spectrum of the simulated signals under different SNR cases.
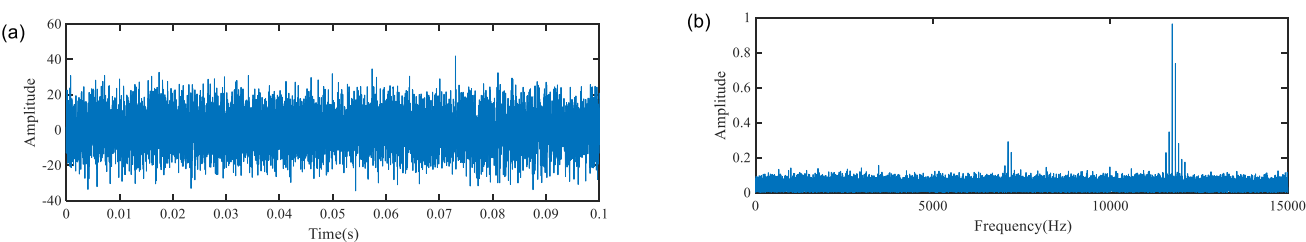

Fig. 10. Simulated signal of an outer race fault: (a) waveform (b) spectrum.

The simulated signals were then processed by the AR model filter. The order range is limited to 100, and the optimal AR model order and its corresponding maximum kurtosis value for the three SNR cases are 8 and 3.027, 7 and 3.042, 9 and 3.024, respectively.

(a)

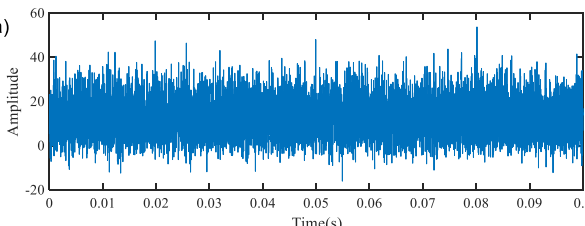

(b)
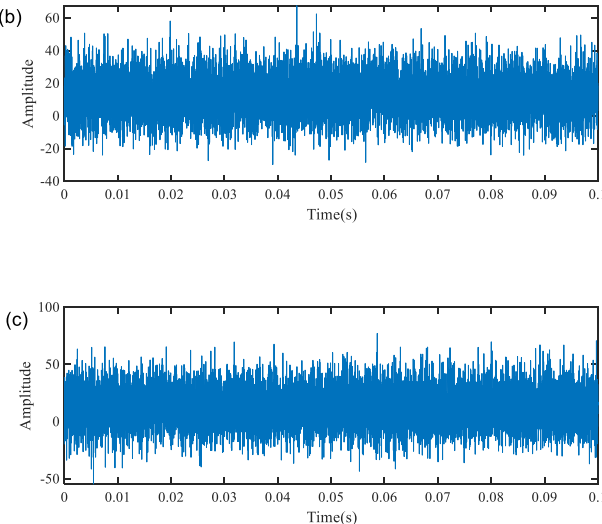
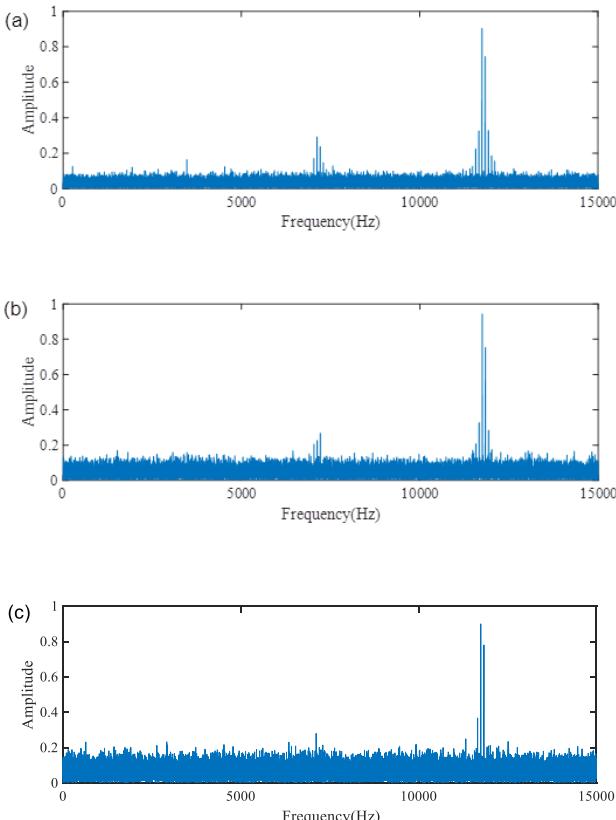

Fig. 11. Waveform and spectrum of the simulated signal with Gaussian noise for different SNRs: 
(a) $\mathrm{SNR}=-6.62 \mathrm{~dB}$, (b) SNR $=-10.43 \mathrm{~dB}$, and (c) $\mathrm{SNR}=-14.40 \mathrm{~dB}$.

To suppress the Gaussian noise and enhance the modulation effect of the simulated signal for bearing outer race fault detection, the MSB was applied to analyze the AR model filtered signal, and the analysis results are plotted in Fig. 12. It was obvious that as the Gaussian noise level increases, the extracted fault features by the proposed AR-MSB were very similar, indicating that the MSB can suppress Gaussian noise effectively.
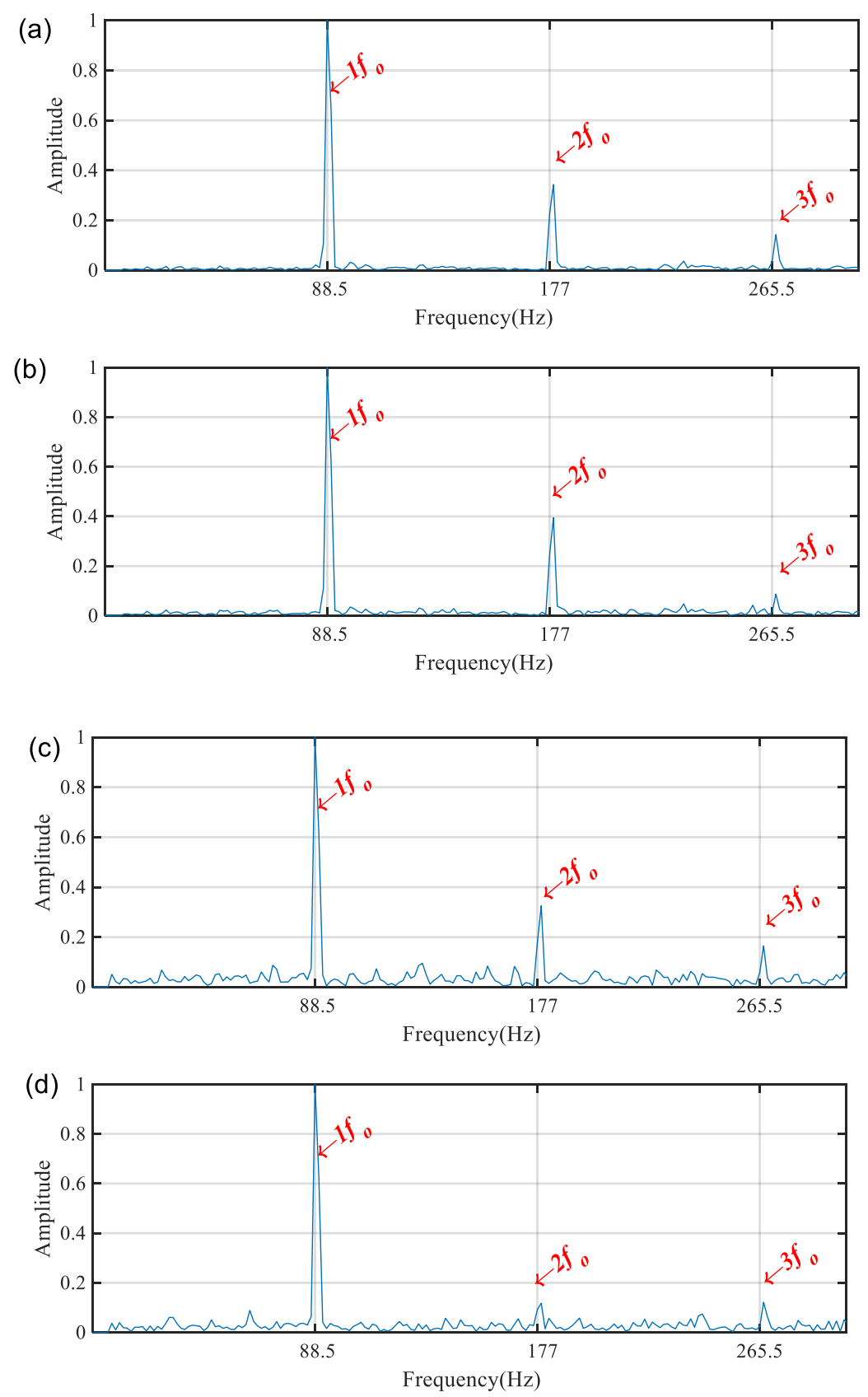

Fig. 12. The analysis result of $A R-M S B$ for Gaussian noise reduction under different SNRs: (a) $\mathrm{SNR}=0 \mathrm{~dB}$, (b) $\mathrm{SNR}=-6.62 \mathrm{~dB}$, (c) $\mathrm{SNR}=-10.43 \mathrm{~dB}$, and (d) $\mathrm{SNR}=-14.40 \mathrm{~dB}$.

\section{Experimental validation}

To investigate the effectiveness of the proposed AR-MSB method for fault feature extraction through suppressing both the Gaussian and non-Gaussian noise, two rolling element bearing 
experimental fault cases were carried out. Additionally, to illustrate the excellence of the AR-MSB method, the analysis results of the AR-MSB were compared with the analysis results using the FK and conventional MSB.

\subsection{Case 1: Fault detection for a planetary gearbox bearing inner race 5.1.1 Experimental setup}

To demonstrate the AR-MSB method, the vibration signal of a deep groove ball bearing with inner race faults was measured for studies. The planetary gearbox experimental device and its photograph were illustrated in Fig. 13. The experimental device was comprised of a DC generator load, DC drive motor, helical gearbox, planetary gearbox and vibration sensors. An inner race fault was seeded on the test bearing as shown in Fig. 14. The structure parameters of the test bearing were shown in Table 1. The piezoelectric accelerometer with a sensitivity of $28.7 \mathrm{mV} / \mathrm{ms}^{-2}$, was installed in the outer housing of the ring gear along the position of the test planetary gearbox bearing to collect the vibration signal. The sampling frequency of the collected data and the rotation of the bearing were $96 \mathrm{kHz}$ and around 1500 $\mathrm{rpm}$, respectively. Therefore, the rotational frequency $f_{r}$ of the shaft is around $25 \mathrm{~Hz}$, and inner race fault characteristic frequency $f_{i}$ is $65.17 \mathrm{~Hz}$.

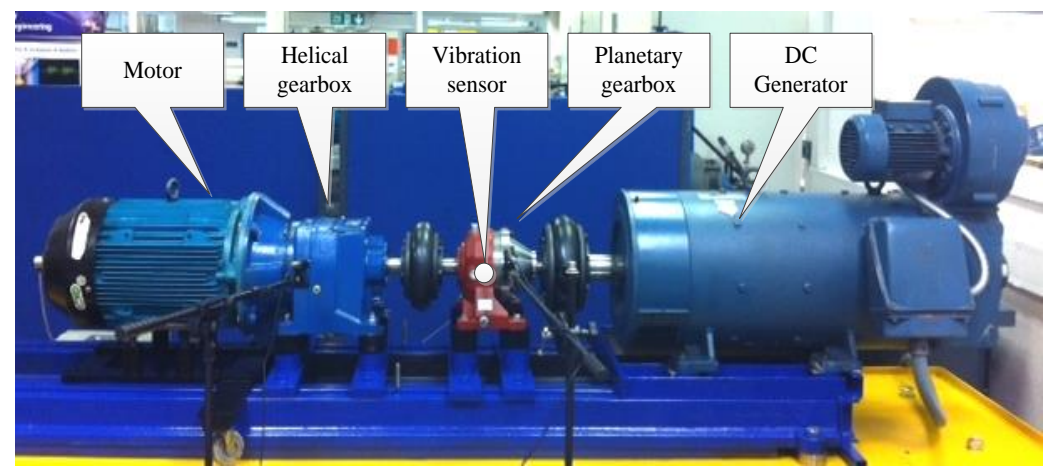

Fig. 13. Planetary gearbox experimental device.

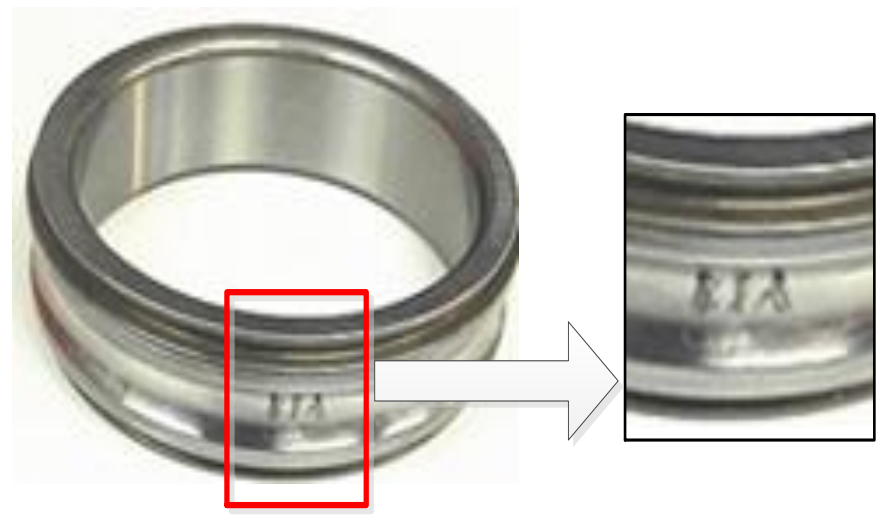

Fig. 14. Inner race fault of the test bearing.

Table 1. The structural parameters of the test bearing.

\begin{tabular}{ccccc}
\hline Bearing model & $\begin{array}{c}\text { Ball diameter } d \\
(\mathrm{~mm})\end{array}$ & $\begin{array}{c}\text { Pitch diameter } D_{m} \\
(\mathrm{~mm})\end{array}$ & Ball number $z$ & Contact angle $\beta$ \\
\hline SKF 6008 & 7.9 & 54 & 12 & $0^{\circ}$ \\
\hline
\end{tabular}

\subsubsection{Detection results for the bearing inner race fault}

Fig. 15(a) and (b) show the waveform and spectrum of the measured vibration signal, respectively. 
It is seen that the measured signal has strong background noise and high modulation characteristics. And it is difficult to identify the fault characteristic frequencies from the spectrum analysis as shown in Fig. 15(b).
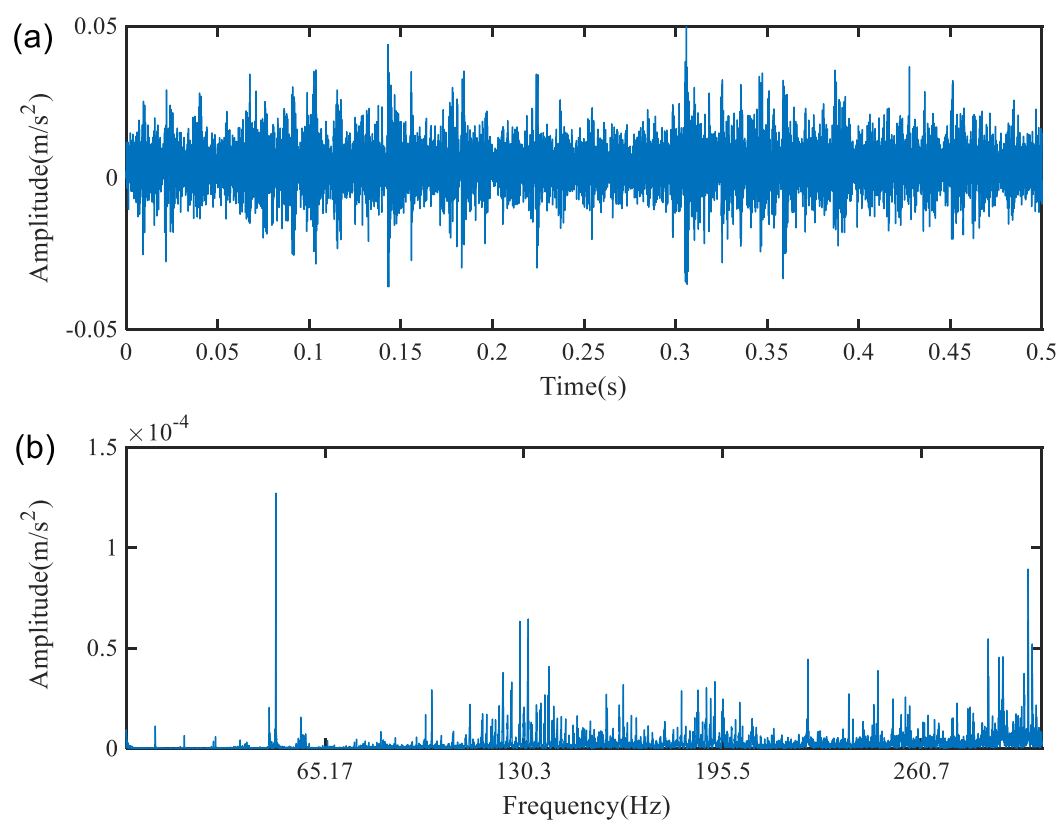

\section{Fig. 15. Waveform and spectrum of the vibration signal under bearing inner race fault:}

\section{(a) waveform (b) spectrum.}

The AR-MSB method was then applied to analyze the vibration signal of the planetary gearbox bearing with inner race fault. The signal was first processed by the AR model filter. The order range was limited to 100, and the optimal AR model order and its corresponding maximum kurtosis value were 2 and 5.02, respectively, as shown in Fig. 16. The spectrum of the AR model filtered signal was depicted in Fig. 17, it cannot present the fault characteristic frequency $f_{i}$ and its harmonics due to strong background noise. The MSB was then applied to analyse the AR model filtered signal to extract the fault features, the result of which is illustrated in Fig. 18(c). It is clearly seen that the AR-MSB method evidently recognizes the inner race fault characteristic frequency $f_{i}$ and its harmonics. Additionally, FK [32] and the conventional MSB [16] that without the preprocessing of the AR model filter, were also used to analyze the measured vibration signal as shown in Fig. 15(a) for comparison. Fig. 18(a) and (b) show the spectrum of the analysis results by conventional MSB and FK, respectively. The spectrum of the FK is mixed with much heavy noise as shown in Fig. 18(b), while conventional MSB analysis results show the effect of harmonics still exists as shown in Fig. 18(a). It demonstrated that the AR-MSB method is able to obtain more accurate results in the detection of a planetary gearbox bearing fault.

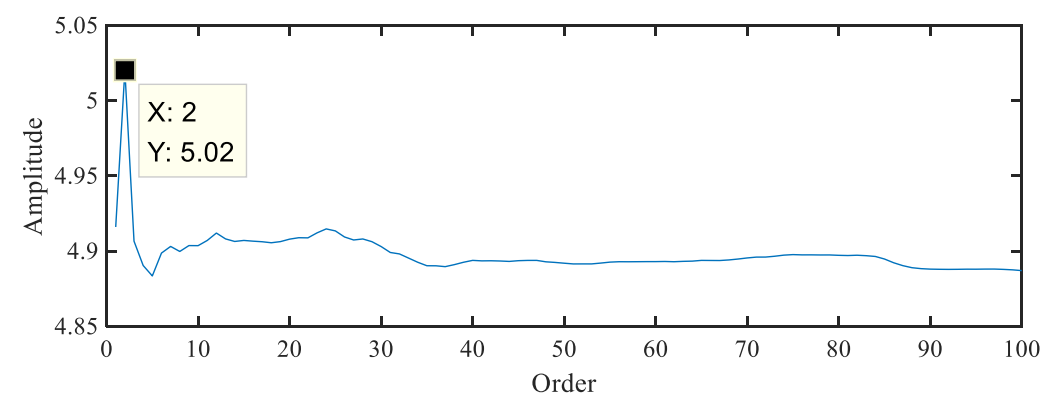




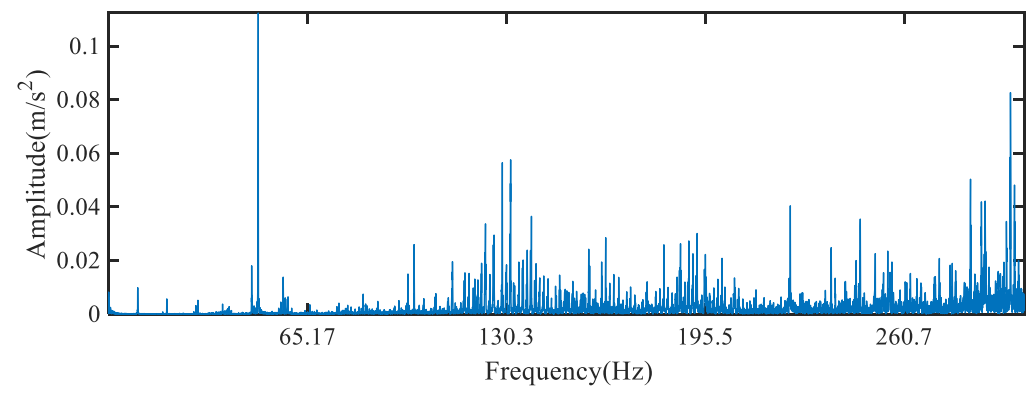

Fig. 17. The spectrum of the AR model filtered signal._
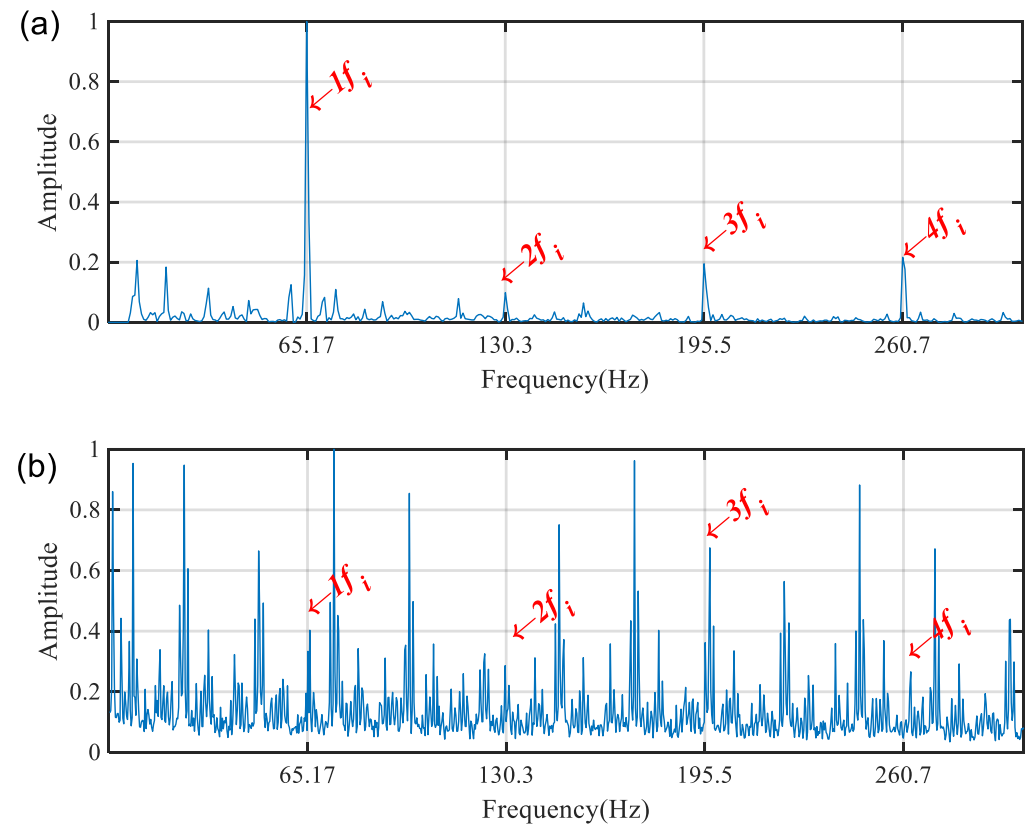

(c)

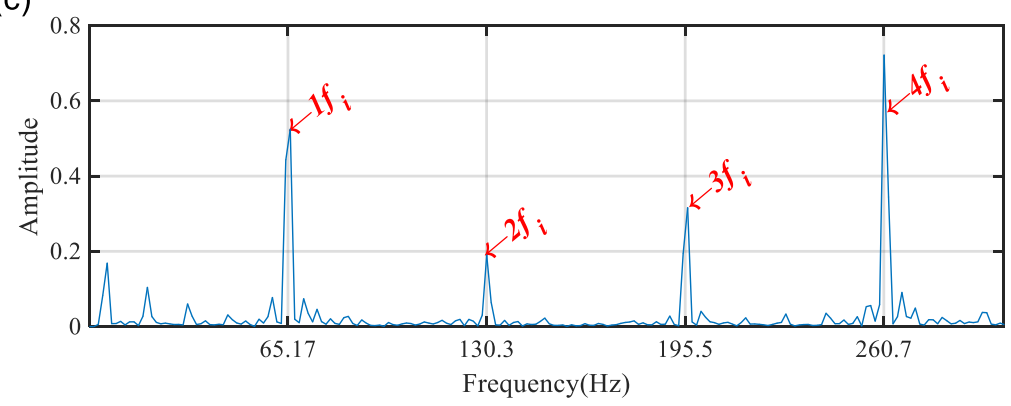

Fig. 18. Analysis results of the vibration signal for bearing inner race fault using: (a) conventional MSB; (b) FK; (c) AR-MSB method.

\subsection{Case 2: Fault detection for a motor bearing outer race}

\subsubsection{Experimental setup}

To validate the proposed AR-MSB again, the experimental vibration signal of the deep groove ball bearing with outer race faults mounted on an induction motor was measured and considered for the proposed method evaluation. The vibration signal was recorded using the bearing test rig illustrated in Fig. 19. The test rig mainly comprised an AC motor, dynamic brake, vibration sensor as well as two flexible coupling, supporting bearings. An outer race fault was seeded on the test bearing as illustrated 
in Fig. 20. The structure parameters of the motor bearing were shown in Table 2. Vibration signals were obtained via a piezoelectric accelerometer, which was installed in the vertical direction on the motor drive end bearing housing with sensitivity of $1.04 \mathrm{mV} / \mathrm{ms}^{2}$. With the sampling frequency of $96 \mathrm{kHz}$, the vibration signal of the motor bearing with outer race fault was collected at a shaft rotating speed around $1500 \mathrm{rpm}$. The $f_{r}$ is $25 \mathrm{~Hz}$ and outer race fault characteristic frequency $f_{o}$ is $89.33 \mathrm{~Hz}$.

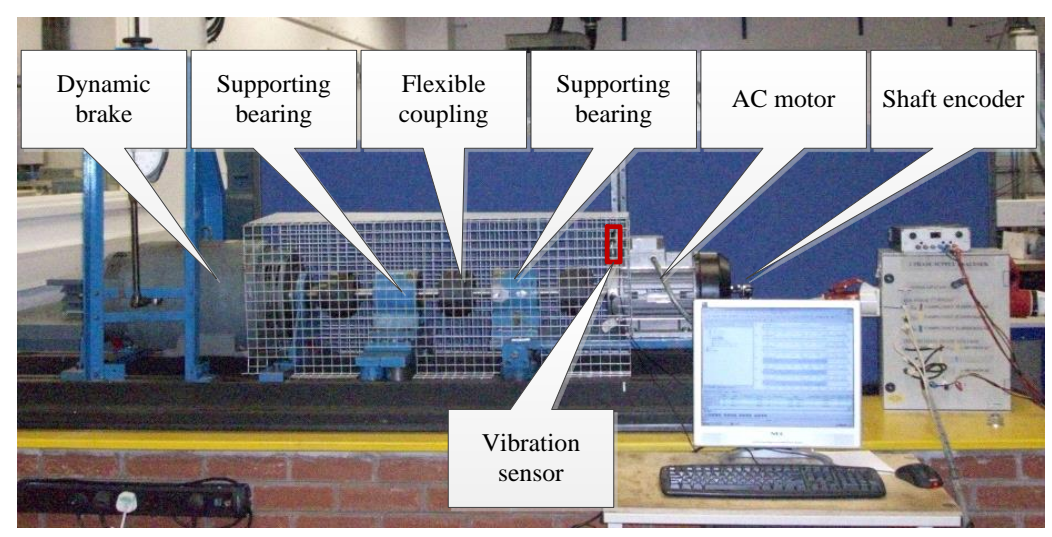

Fig. 19. Motor bearing experimental device.
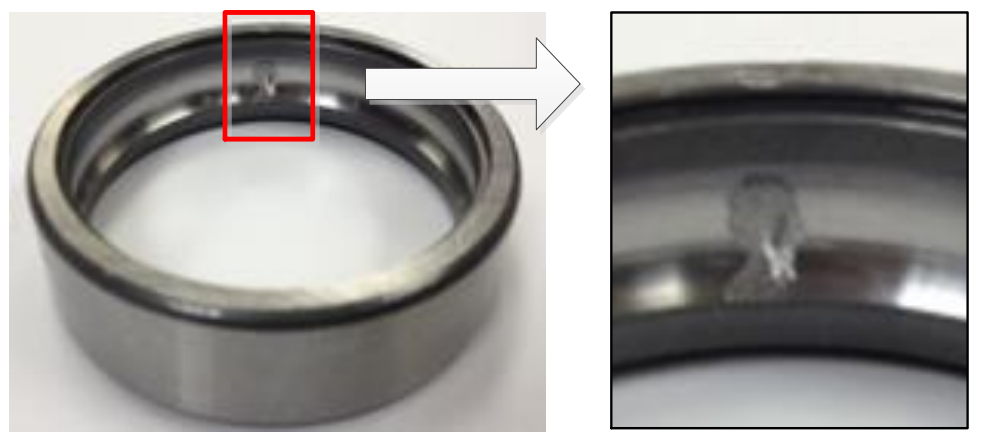

Fig. 20. Outer race fault of motor bearing.

Table 2. Structural parameters of the motor bearing.

\begin{tabular}{ccccc}
\hline Bearing model & $\begin{array}{c}\text { Ball diameter } d \\
(\mathrm{~mm})\end{array}$ & $\begin{array}{c}\text { Pitch diameter } D_{m} \\
(\mathrm{~mm})\end{array}$ & Ball number $z$ & Contact angle $\beta$ \\
\hline $6206 \mathrm{ZZ}$ & 9.53 & 46.4 & 9 & $0^{\circ}$ \\
\hline
\end{tabular}

\subsubsection{Detection results for the outer race of the motor bearing}

Fig. 21 illustrates the waveform and spectrum of the vibration signal of the tested motor bearing with outer race fault. From Fig. 21(b), it can be seen that it's higher order harmonics (i.e. $2 f_{o}$ and $3 f_{o}$ ), but the fault characteristic frequency $f_{o}$ cannot be observed under strong background noise. 

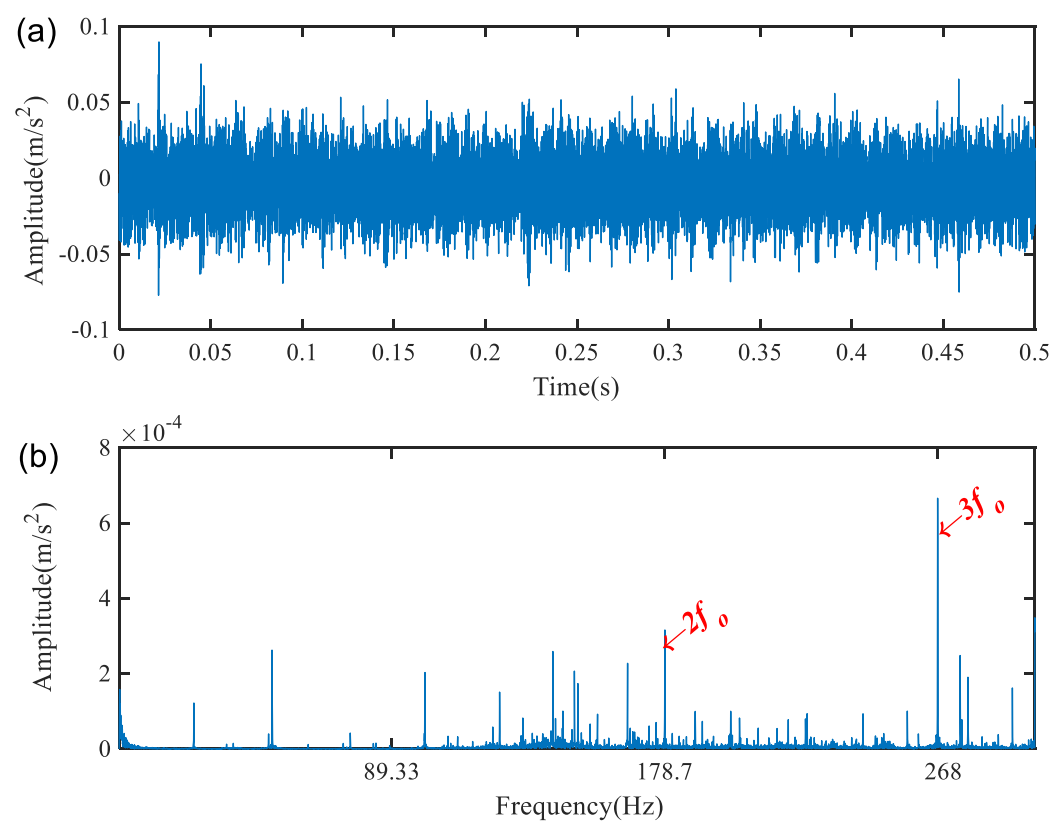

Fig. 21. Waveform and spectrum of the measured vibration signal for motor bearing outer race fault: (a) waveform (b) spectrum.

The AR-MSB method was then employed to analyze the vibration signal shown in Fig. 21 (a). The signal was processed using the AR model filter firstly. The order range was limited to 100, and the optimal AR model order and its corresponding maximum kurtosis value were 5 and 3.279 as shown in Fig. 22. The spectrum of the AR model filtered signal was shown in Fig. 23. It can be seen that only the higher order harmonics (i.e. $2 f_{o}$ and $3 f_{o}$ ) can be recognized, but the fault characteristic frequency $f_{o}$ cannot be identified. To suppress Gaussian noise and strengthen the modulation effects of the motor bearing fault, the MSB was applied to analyze the AR model filtered signal, and the results are illustrated in Fig. 24(c). For comparison purpose, the FK and the conventional MSB were also applied to extract fault characteristic frequency based on the analysis of the measured vibration signals shown in Fig. 21 (a), the results of which were plotted in Fig. 24(a) and (b), respectively. Even the fault characteristic information can be identified, but the FK analysis results are corrupted with much heavy noise as shown in Fig. 24(b). The conventional MSB reflected the fault characteristic frequency $f_{o}$ and its harmonics, but there are still interfering frequencies, particularly around the higher-frequency harmonics as depicted in Fig. 24(a). By contrast, Fig. 24(c) clearly shows that the AR-MSB method can distinctly recognize the outer race fault characteristic frequency $f_{o}$ and its harmonics. Hence it can indicate that the AR-MSB method can extract fault characteristic frequencies well and reduces noise greatly.

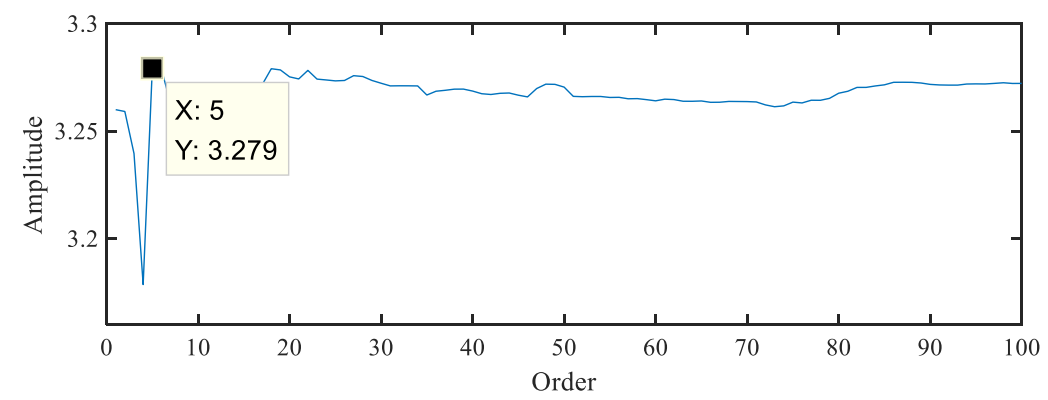


Fig. 22. Optimum AR model order and the related maximum kurtosis.

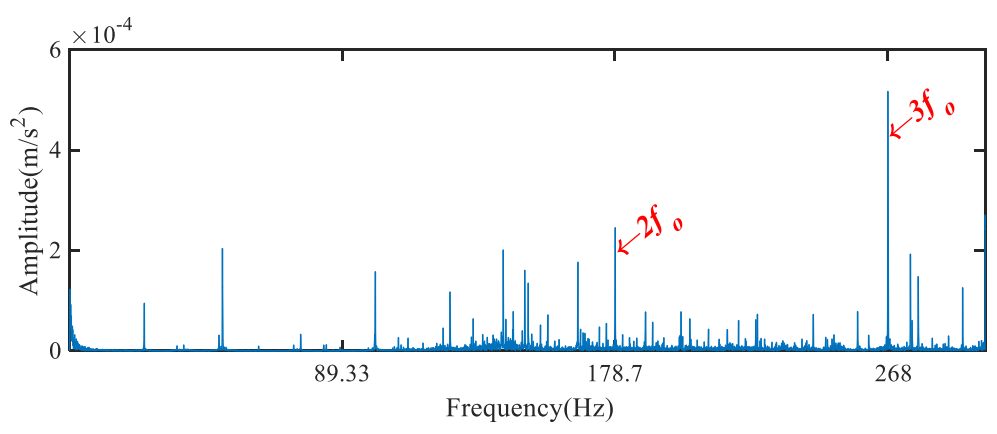

Fig. 23. The spectrum of the AR model filtered signal.
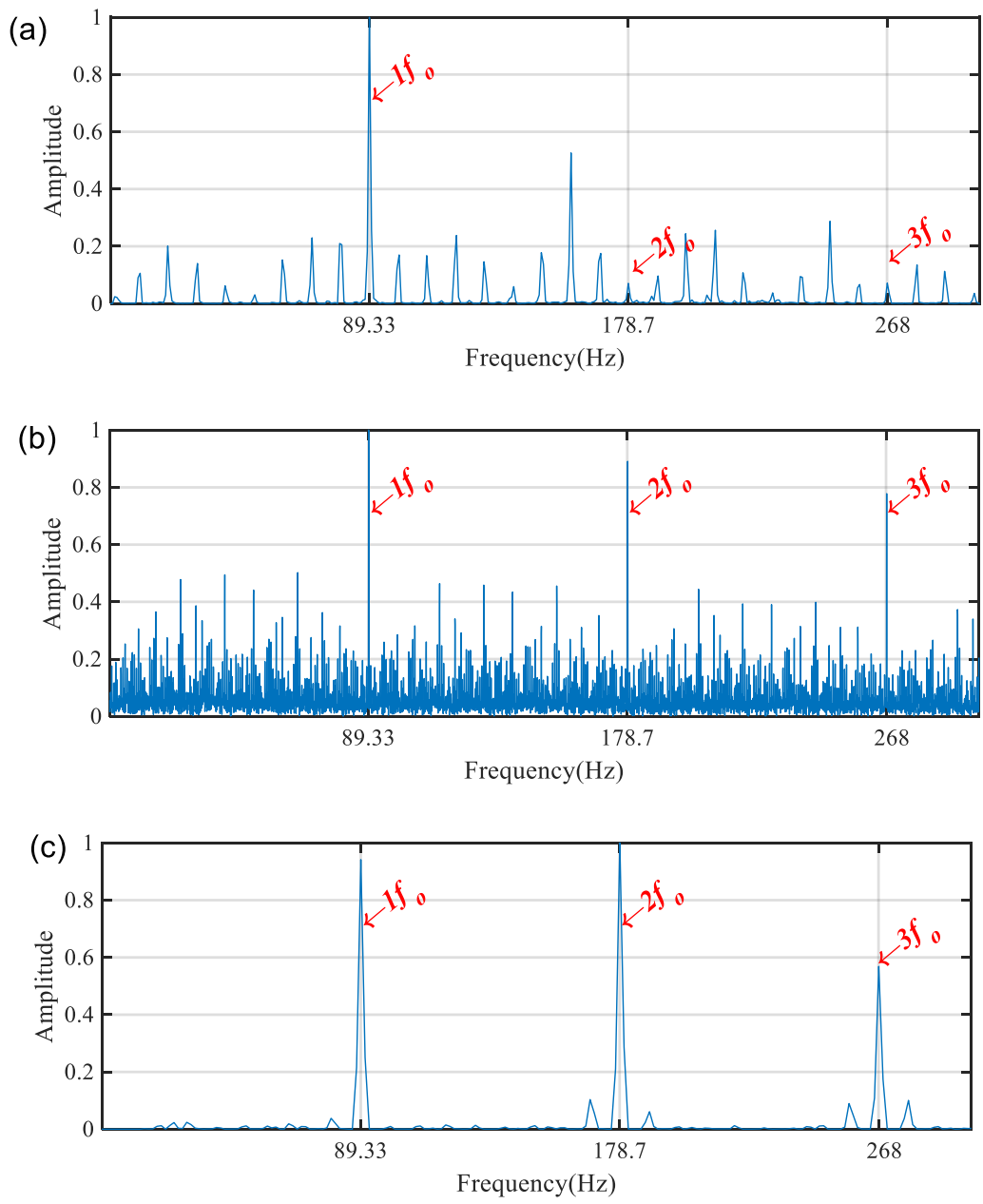

Fig. 24. Analysis results for the motor bearing outer race fault feature extraction using:

(a) conventional MSB (b) FK and (c) AR-MSB.

\section{Conclusion}

This paper proposed a novel methodology for rolling element bearing fault detection based on an enhanced MSB analysis by making use of AR model filter. The proposed method combined the merits of the AR model as the pre-filter to reduce non-Gaussian noise, and the MSB suppresses Gaussian noise and decomposes the modulated components for accurate fault feature extraction. The proposed methodology was then verified via simulation signals and experimental case studies. The results 
presented that the AR-MSB method provided more exact detection results with high spectral resolution and reduced the impulsive interference of MSB analysis results, thus improving the performance of MSB effectively. In addition, the results shown that the AR-MSB approach have higher accuracy in fault feature extraction than the conventional MSB and FK. Although the proposed method was only applied to the fault detection of rolling element bearing, it can be extended to be used for fault feature extraction and detection of many other rotating machines, such as gearbox, motor and compressor, etc. Additionally, the proposed AR-MSB method needs to be developed to handle the compound faults or other rotating mechanical faults detection under variable speed conditions in the future work.

\section{Acknowledgements}

The research work was supported by the National Natural Science Foundation of China (grant numbers 51605133, 51705127), and the Hebei Provincial International Science and Technology Cooperation Program of China (grant number 17394303D).

\section{References}

[1] N.A. Khan, M. Sandsten, Time-frequency image enhancement based on interference suppression in Wigner-Ville distribution, Signal. Processing. 127 (2016) 80-85.

[2] C. Mateo, J.A. Talavera, Short-Time fourier transform with the window size fixed in the frequency domain, Digit. Signal. Process. 77 (2018) 13-21.

[3] Y. Xu, Z. Cai, K. Ding, An enhanced bearing fault diagnosis method based on TVF-EMD and a highorder energy operator, Meas. Sci. Technol. 29 (2018) 095108.

[4] Z. Duan, T. Wu, S. Guo, et al., Development and trend of condition monitoring and fault diagnosis of multi-sensors information fusion for rolling bearings: a review, Int. J. Adv. Manuf. Tech. 96 (1-4) (2018) 803-819.

[5] W. Caesarendra, T. Tjahjowidodo. A review of feature extraction methods in vibration-based condition monitoring and its application for degradation trend estimation of low-speed slew bearing, Machines, 5 (21) (2017) 1-28.

[6] Z. Feng, Y. Zhou, M. Zuo, et al. Atomic decomposition and sparse representation for complex signal analysis in machinery fault diagnosis: A review with examples. Measurement. 103 (2017) 106-132.

[7] M. Kang, J. Kim, L.M. Wills, et al., Time-Varying and multiresolution envelope analysis and discriminative feature analysis for bearing fault diagnosis, IEEE. Trans. Ind. Electron. 62 (12) (2015) 7749-7761.

[8] J. Wang, Q. He, Wavelet packet envelope manifold for fault diagnosis of rolling element bearings, IEEE. Trans. Instrum. Meas. 65 (11) (2016) 2515-2526.

[9] Jérôme Antoni, Fast computation of the kurtogram for the detection of transient faults, Mech. Syst. Signal. Process. 21 (1) (2007) 108-124.

[10] M. Gao, G, Yu, T. Wang, Impulsive gear fault diagnosis using adaptive morlet wavelet filter based on alpha-stable distribution and Kurtogram. IEEE. Access. 7 (2019) 72283-72296.

[11] Y. Wang, P.W. Tse, B. Tang, et al., Kurtogram manifold learning and its application to rolling bearing weak signal detection, Measurement. 127 (2018) 533-545.

[12] S. Lu, X. Wang, F. Liu, et al. Fault diagnosis of motor bearing by analyzing a video clip, Math. Probl. Eng. http://dx.doi.org/10.1155/2016/8139273.

[13] T. Wang, F. Chu, Z. Feng, Meshing frequency modulation (MFM) index-based kurtogram for planet bearing fault detection, J. Sound. Vib. 432 (2018) 437-453. 
[14] F. Gu, Y. Shao, N. Hu, et al. Electrical motor current signal analysis using a modified bispectrum for fault diagnosis of downstream mechanical equipment, Mech. Syst. Signal. Process. 25 (1) (2011) 360-372.

[15] R. Zhang, F. Gu, H. Mansaf, et al., Gear wear monitoring by modulation signal bispectrum based on motor current signal analysis, Mech. Syst. Signal Process. 94 (2017) 202-213.

[16] X. Tian, J.X. Gu, I. Rehab, et al., A robust detector for rolling element bearing condition monitoring based on the modulation signal bispectrum and its performance evaluation against the Kurtogram, Mech. Syst. Signal. Process. 100 (1) (2018) 167-187.

[17] J. Guo, Z. Shi, H. Li, et al., Early fault diagnosis for planetary gearbox based wavelet packet energy and modulation signal bispectrum analysis, Sensors. 18 (2018) 2908.

[18] J. Guo, D. Zhen, H. Li, et al., Fault feature extraction for rolling element bearing diagnosis based on a multi-stage noise reduction method, Measurement. 139 (2019) 226-235.

[19] X. Song, S. Wang, Z. Hu, et al., A hybrid Rayleigh and Weibull distribution model for the short-term motion response prediction of moored floating structures, Ocean Engineering. 182 (2019) 126-136.

[20] W. Xu, W. Chen, Y. Liang, Feasibility study on the least square method for fitting non-Gaussian noise data, Physica A. 492 (2018) 1917-1930.

[21] Y. Guo, Y. Shen, J. Tan, Stochastic resonance in a piecewise nonlinear model driven by multiplicative non-Gaussian noise and additive white noise, Commun. Nonlinear. Sci. 38 (2016) 257266.

[22] G. Wang, N. Li, Y. Zhang, Maximum correntropy unscented Kalman and information filters for nonGaussian measurement noise, J. Franklin. I. 354 (2017) 8659-8677.

[23] H. Asadi, B. Seyfe, Signal enumeration in Gaussian and non-Gaussian noise using entropy estimation of eigenvalues, Digit. Signal. Process. 78 (2018) 163-174.

[24] L.K. Yen, J.C. Principe, Weak transient signal detection in non-Gaussian noise using RBF and recurrent networks, Proceedings of SPIE-The International Society for Optical Engineering. 3395 (1998) 69-75.

[25] C.K. Kovach, H. Oya, H. Kawasaki. The bispectrum and its relationship to phase-amplitude coupling, NeuroImage, 173 (2018) 518-539.

[26] T. Zhang, W. Chen, M. Li. AR based quadratic feature extraction in the VMD domain for the automated seizure detection of EEG using random forest classifier, Biomed. Signal. Proces. 31 (2017) 550-559.

[27] C.C. Wang, Y. Kang, P.C. Shen, et al., Applications of fault diagnosis in rotating machinery by using time series analysis with neural network, Expert. Syst. Appl. 37 (2010) 1696-1702.

[28] A. Datteo, F. Loca, G. Busca, Statistical pattern recognition approach for long-time monitoring of the G.Meazza stadium by means of AR models and PCA, Eng. Struct. 153 (2017) 317-333.

[29] P. Saha, S. Ghorai, B. Tudu, et al., Tea quality prediction by autoregressive modeling of electronic tongue signals, IEEE. Sens. J. 16 (2016) 4470-4477.

[30] A. Datteo, G. Busca, G. Quattromani, et al., On the use of AR models for SHM: A global sensitivity and uncertainty analysis framework, Reliab. Eng. Syst. Safe. 170 (2018) 99-115.

[31] M. Shannon, H. Zen, W. Byrne, Autoregressive models for statistical parametric speech synthesis, IEEE. Trans. Audio. Speech. 21 (2013) 587-597.

[32] F. Cong, J. Chen, G. Dong, Spectral kurtosis based on AR model for fault diagnosis and condition monitoring of rolling bearing, J. Mech. Sci. Technol. 26 (2012) 301-306.

[33] H. Al-Bugharbee, I. Trendafilova, A fault diagnosis methodology for rolling element bearings based 
on advanced signal pretreatment and autoregressive modelling, J. Sound. Vib. 369 (2016) 246-265.

[34] H. Ogasawara, Bias correction of the Akaike information criterion in factor analysis, J. Multivariate. Anal. 149 (2016) 144-159.

[35] A. Zanini, A.D. Woodbury, Contaminant source reconstruction by empirical Bayes and Akaike's Bayesian information criterion, J. Contam. Hydrol. 185-186 (2016) 74-86.

[36] W.D. Penny, S.J. Roberts, Bayesian method for autoregressive models. in: Proc. IEEE Int. Workshop Neural Networks Signal Process 2000.

[37] X. Wang, V. Makis, Autoregressive model-based gear shaft fault diagnosis using the KolmogorovSmirnov test, J. Sound. Vib. 327 (2009) 413-423.

[38] L. Shi, J. Shen, Y. Zhang, et al., Fault diagnosis of a rolling element bearing based on AR model and spectral kurtosis, J. Vibration. Shock. 30 (2011) 257-260.

[39] Z. Zhang, W. Gui, Statistical inference of reliability of generalized Rayleigh distribution under progressively type-II censoring, Journal of Computational and Applied Mathematics. 361 (2019) 295312.

[40] H. Zhu, X. Pan, Q. Xie, Merging Student's-t and Rayleigh distributions regression mixture model for clustering time-series, Neurocomputing. 266 (2017) 247-262. 